\title{
Macrophages and MHC class II positive cells in the choroid during endotoxin induced uveitis
}

\author{
Peizeng Yang, Alex F de Vos, Aize Kijlstra
}

Department of OphthalmoImmunology, the Netherlands Ophthalmic Research Institute, Amsterdam, the Netherlands P Yang

A F de Vos

A Kijlstra

\section{Zhongshan} Ophthalmic Centre, Guangzhou, PR China P Yang

Department of Ophthalmology, University of Amsterdam, the Netherlands A Kijlstra

Correspondence to: A Kijlstra, Netherland Ophthalmic Research Institute, Department of Ophthalmo-Immunology, PO Box 12141, 1100 AC Amsterdam, Netherlands.

Accepted for publication 12 February 1997

\begin{abstract}
Aims/background-Endotoxin induced uveitis has been regarded as a model for acute anterior uveitis and until now little was known about choroidal involvement. The aim of this study was to investigate changes in macrophages and MHC class II positive cells in the choroid of Lewis rats during endotoxin induced uveitis.

Methods-Choroid-sclera wholemounts were isolated from normal Lewis rats and at different time points- $4,8,16,24,48,72$, and 96 hours, and 7, 10, and 14 days after a footpad injection of $200 \mu \mathrm{g}$ of lipopolysaccharide (LPS). Immunohistochemistry was performed using the monoclonal antibodies ED1 (monocytes, macrophages, dendritic cells), and OX6 (MHC class II antigen).

Results-In normal rats, two layers of macrophages were identified in the choroid; a layer located immediately beneath the retinal pigment epithelium (RPE) and a layer bordering the sclera. The density of ED1 positive cells in the layer bordering the RPE cells was 902 (SD 132) cells $/ \mathrm{mm}^{2}$ whereas the scleral layer had a cell density of 389 (73) cells $/ \mathrm{mm}^{2}$. Based on morphology, positive cells could be divided into two main categories; pleomorphic/round cells and dendritiform cells with varying appearances, with the latter being predominant in normal eyes. A network of MHC class II positive dendritic cells was found in the choroid, beneath the RPE, with a density of 659 (96) cells $/ \mathrm{mm}^{2}$. No MHC class II positive cells were found in the macrophage layer bordering the sclera. LPS injection caused a massive influx of ED1 positive macrophages in the area below the RPE cells but did not result in an influx of macrophages at the scleral side of the choroid. The infiltrate reached a maximum at 16 hours following LPS injection and decreased at 96 hours. The morphology of the infiltrating cells was pleomorphic/round at early stages of inflammation and changed into a dendritiform cell population later. The number of MHC class II positive cells on the anterior side of the choroid increased 8 hours after injection and reached a peak at 72-96 hours. MHC class II positive cells were not observed in the vicinity of the sclera at any time after LPS injection. Both resident and MHC class II positive dendritic cell numbers returned to nor-
\end{abstract}

mal values at day 14 following LPS injection.

Conclusions-These results indicate that the choroid is severely inflamed after systemic LPS administration to Lewis rats and suggests that endotoxin induced uveitis may serve as a model for generalised uveitis in humans.

(Br f Ophthalmol 1997;81:396-401)

Numerous animal models have been set up to study the pathogenesis of uveitis. ${ }^{1}$ These include models of autoimmune posterior uveitis whereby a number of antigens such as rhodopsin, phosducin, recoverin, $S$ antigen, and interphotoreceptor retinoid binding protein, are capable of inducing retinal autoimmune disease in a number of laboratory animals including mice, guinea pigs, rats, and primates. Autoimmune anterior uveitis can be induced in experimental animals by using melanin associated protein. These animal models have revealed that autoimmune uveitis is a $\mathrm{T}$ cell mediated disease which can be effectively treated by suppressing $\mathrm{T}$ cell functions. ${ }^{1}$ Another widely used animal (rat, mouse) model for uveitis involves the systemic (footpad) administration of endotoxin, which may serve as a tool to study uveitis associated with bacterial infections occurring elsewhere in the body. ${ }^{2}$ Endotoxin induced uveitis (EIU) is characterised by iris hyperaemia, miosis, increased protein and fibrin in the anterior chamber, and massive cell infiltration in the iris and ciliary body. ${ }^{2}$ In view of the immense inflammation of the anterior segment this model has always been considered a representative of anterior uveitis. Recently, it has become apparent that the posterior segment of the eye may also be involved in the EIU model. Using whole mounts of the retina we recently reported the involvement of the retina during EIU whereby it was noted that lipopolysaccharide (LPS) injection was followed by a massive influx of macrophages in the retina. ${ }^{3}$ A hallmark was the absence of MHC class II positive cells in the retina before and after LPS injection.

In the present study we focused our attention to the choroid. In contrast with the retina, the normal choroid already contained a network of MHC class II positive dendritic cells. Following systemic LPS injection, the choroid became heavily infiltrated with monocytes, macrophages, and MHC class II positive cells. The severe inflammation of both the retina and choroid during EIU indicates that, 

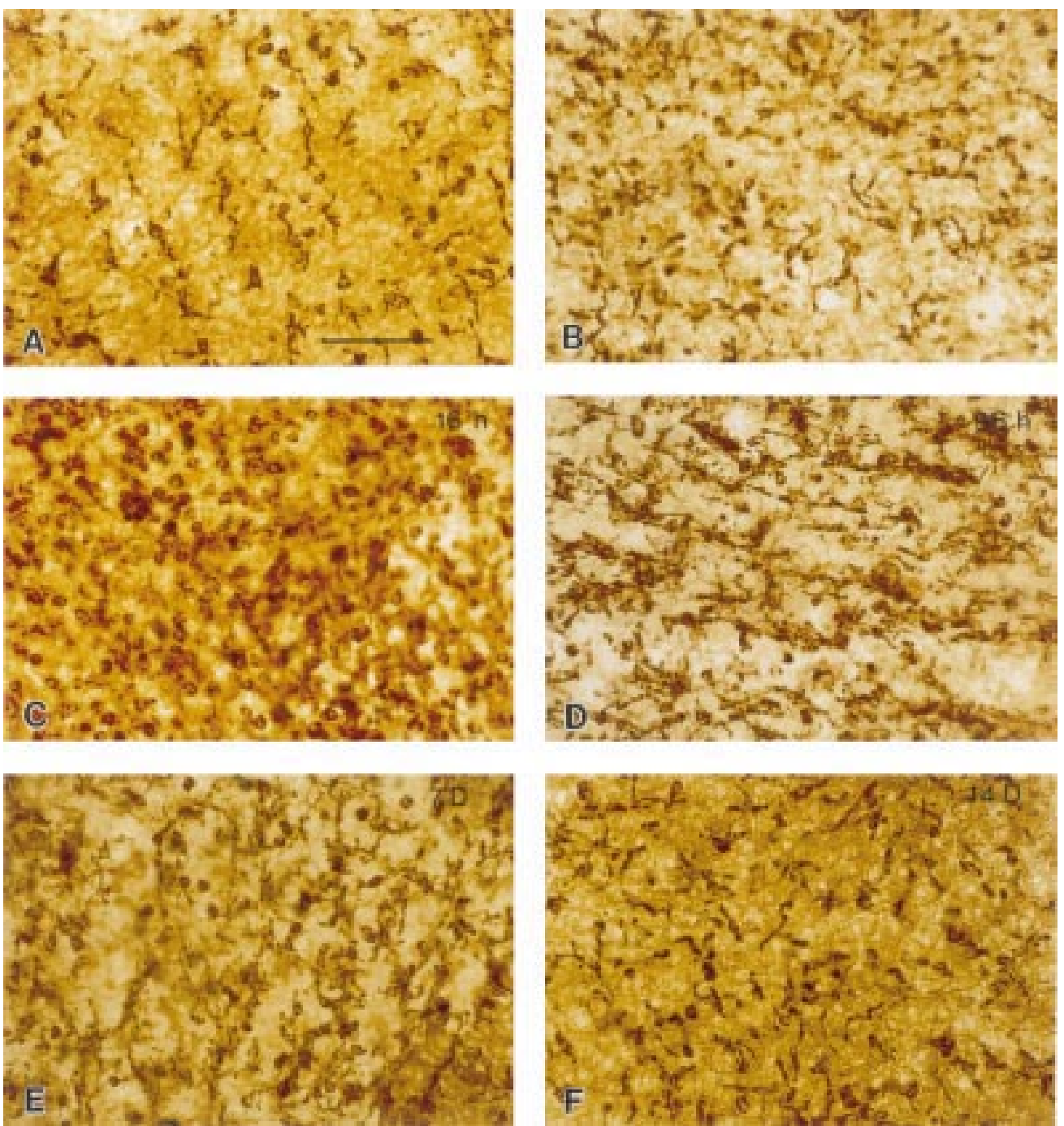

Figure 1 Immunohistochemical staining of choroidal wholemounts with monoclonal antibody ED1. Wholemounts were obtained from normal Lewis rats $(A, B)$ and at different time points after footpad lipopolysaccharide injection: 16 hours $(C), 96$ hours $(D)$, day $7(E)$, and day $14(F)$. In normal rats, ED1 positive cells were present in the inner ' $R P E$ bordering'layer $(A)$ and an outer layer adjacent to the sclera $(B)$. Note the presence of ED1 positive cells after the RPE layer was brushed off $(E)$; the remaining RPE is still detectable as faintly stained hexagonal cells in the right hand bottom corner $(E)$. Magnification bar in $(A)$ equals $250 \mu \mathrm{m}$ and refers to all parts of the figure.

although anterior uveitis is a very prominent aspect of this disorder, EIU may also be used as an experimental model of panuveitis.

\section{Materials and methods}

EXPERIMENTAL DESIGN

Fifty inbred male Lewis rats (6-8 weeks of age), weighing 150-200 g, were used in the study. All animals were treated according to the ARVO statement for the use of animals in ophthalmic and vision research. LPS (Salmonella typhimurium, Difco Ltd, Detroit, MI, USA) was dissolved in sterile pyrogen-free saline $0.9 \%$ at a concentration of $2 \mathrm{mg} / \mathrm{ml}$. Fifty $\mu \mathrm{l}$ of LPS was injected into each hind footpad of Lewis rats, the total doses per animal being 200 $\mu \mathrm{g}$. At different time points-for example, before LPS injection (control group), and 4, 8, $16,24,48,72$, and 96 hours, and 7, 10, and 14 days after LPS injection, rats were perfused and whole mounts of the choroid-sclera complex were prepared as described below. The number of Lewis rats in each group varied between 2 and 6 .
ISOLATION AND PREPARATION OF WHOLE MOUNTS OF THE CHOROID-SCLERA COMPLEX

Rats were anaesthetised by intramuscular injection of $0.5 \mathrm{mg}$ fluanisone and $15 \mu \mathrm{g}$ fentanyl citrate ( $50 \mu$ l of Hypnorm, Janssen, Goirle, the Netherlands) and an intraperitoneal injection of $30 \mathrm{mg}$ sodium pentobarbitone $(0.5 \mathrm{ml}$ Nembutal, Sanofi, Maassluis, the Netherlands). Intravascular coagulation was prevented by injection of $0.3 \mathrm{ml}$ of heparin $(5000$ $\mathrm{IU} / \mathrm{ml}$ ) into the left ventricle. Perfusion through the left ventricle with cold phosphate buffered saline (PBS, pH 7.4) was performed to ensure that blood was completely expelled from the blood vessels. Eyes were immediately enucleated and extraocular tissues were removed. The enucleated eyes were dissected into an anterior and posterior part by an incision behind the ciliary body. Lens and vitreous body were carefully removed from the posterior part. The choroid-sclera complex was gently separated from the retina and subsequently placed in cold $100 \%$ ethanol for 

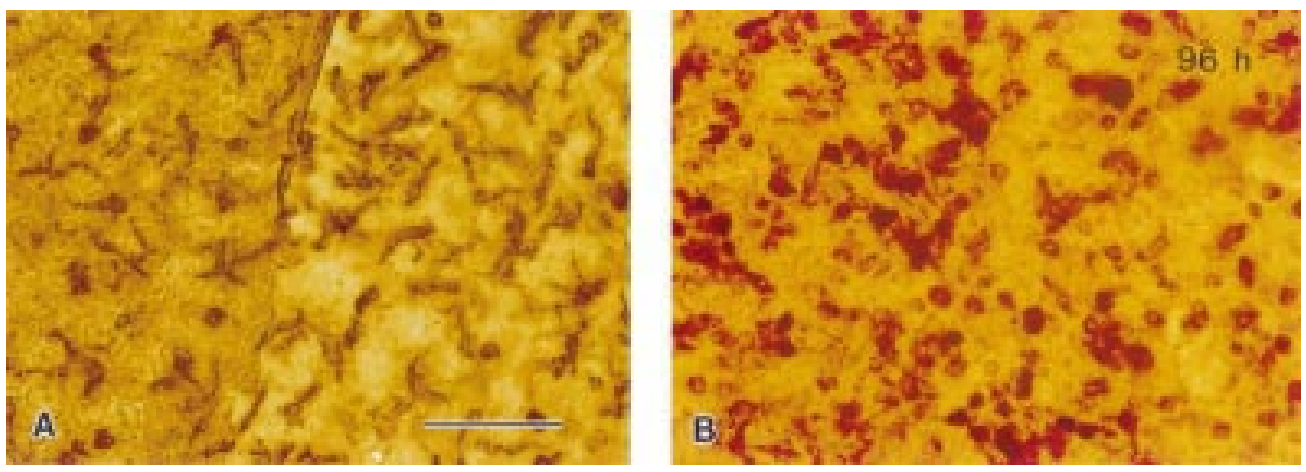

Figure 2 Immunohistochemical staining of choroidal wholemounts with monoclonal antibody OX6 ( $A, B)$. Wholemounts were obtained from a normal Lewis rat $(A)$ and 96 hours after footpad LPS injection $(B)$. Note the presence of OX6 positive cells after partial removal of the RPE layer $(A)$; the RPE layer is still visible as faintly stained hexagonal cells in the right, one third, part of $(A)$. Magnification bar in $(A)$ equals $250 \mu \mathrm{m}$ and refers to both parts of the figure.

fixation for 5 minutes. The fixed choroid-sclera complex was stored at $4^{\circ} \mathrm{C}$ until use.

IMMUNOHISTOCHEMISTRY

Monoclonal antibody ED1 (recognising a cytoplasmic antigen in rat monocytes, macrophages, and $90 \%$ of dendritic cells) was kindly provided by Dr C D Dijkstra, Free University, Amsterdam. ${ }^{4}$ Monoclonal antibody OX6 (recognising rat MHC class II antigen) was purchased from Sera-Lab (Sussex).

Other reagents used were biotinylated sheep anti-mouse antibody (Amersham Life Science, Amersham), streptavidin-biotin-peroxidase complex (Dako, Glostrup, Denmark) and 3,3 diaminobenzidine tetrahydrochloride (DAB; Sigma, St Louis, MO, USA). A standard ABC technique was performed on the whole mounts of the choroid-sclera complex. Endogenous peroxidase activity was eliminated by incubating the tissue in $1 \%$ hydrogen peroxide in PBS for 20 minutes. Wholemounts were incubated with monoclonal antibodies at $4^{\circ} \mathrm{C}$ overnight. All other procedures were performed at room temperature. Stained wholemounts were placed on gelatin coated glass slides with the inner side facing up, dried at room temperature, and embedded in Entellan.

QUANTITATIVE ANALYSIS

Quantitative analysis of ED1 and OX6 positive cells was carried out on normal choroid wholemounts using a calibrated eyepiece graticule under a microscope with $40 \times$ object lens. Different layers of stained cells could be distinguished in separate focal planes of the whole mounts. Immunostained cells were counted in three separate fields per wholemount and the mean number of cells $/ \mathrm{mm}^{2}$ of each rat was calculated. In total, six eyes from six rats were examined and the mean (SEM) determined for the whole group. The data were analysed using Student's $t$ test.

Table 1 Differences in tissue resident macrophages and MHC class II positive cells between the inner 'RPE bordering'layer and the outer layer adjacent to the sclera. (Data represent the mean (SEM))

\begin{tabular}{llll}
\hline Cell type & Inner layer & Outer layer & p Value \\
\hline ED1 positive cells (cells $\left./ \mathrm{mm}^{2}\right)$ & $902(132)$ & $389(73)$ & $<0.01$ \\
Dendritiform (\%) & $74(6)$ & $93(11)$ & $<0.01$ \\
MHC II positive cells (cells $\left./ \mathrm{mm}^{2}\right)$ & $659(96)$ & Absent & \\
Dendritiform (\%) & $83(10)$ & & \\
\hline
\end{tabular}

The number of infiltrating cells in the choroid wholemounts after LPS injection was difficult to quantitate in view of the massive influx of cells and was therefore only performed semiquantitatively. The density of immunopositive cells in the choroid was scored (in a masked manner) as + (the normal levelthat is, low), ++ (mild to moderate increase of cells in some areas), or +++ (a high number of cells in large areas or the whole choroid). Cells were grouped as round/pleomorphic or dendritiform.

\section{Results}

MACROPHAGES AND MHC CLASS II POSITIVE CELLS IN THE NORMAL CHOROID

Analysis of choroidal wholemounts of normal Lewis rats revealed a network of ED1 stained macrophages as well as MHC class II positive cells (ED1, Fig 1: MHC class II, Fig 2). Most of the cells had a dendritic appearance although approximately $25 \%$ had a round or pleomorphic shape. Macrophages were observed in two distinct planes in the wholemounts. One layer was located immediately beneath the RPE, and the other bordered the sclera. Both layers contained ED1 positive macrophages, but the layer in the vicinity of the sclera lacked MHC class II positive cells. The location of these cells beneath the RPE, is illustrated in wholemounts stained with OX6, where part of the RPE cell layer was brushed off (Fig 2A).

Quantitative analysis was performed for ED1 positive cells and showed that the RPE bordering layer (902 (132) cells per $\mathrm{mm}^{2}$ ) contained significantly more macrophages than the scleral side (389 (73) cells per $\mathrm{mm}^{2} ; \mathrm{p}<$ 0.0001 ) (Table 1).

The number of MHC class II positive cells just below the RPE was 659 (96) cells per $\mathrm{mm}^{2}$, and was significantly lower than the number of ED1 positive cells in this layer of the choroid.

CHANGES IN THE CHOROID AFTER LPS INJECTION Systemic LPS administration resulted in a dramatic change in the macrophage and dendritic cell population in the choroid (Figs 1 and 2). The changes occurred mainly in the inner layer at an early stage after LPS injection. ED1 
Table 2 Changes in ED1 positive cells in the choroid following systemic lipopolysaccharide injection

\begin{tabular}{ll}
\hline 4 and 8 hours & $\begin{array}{l}\text { Increased number of round cells, } \\
\text { occasionally accompanied by an } \\
\text { increase of dendritiform cells }\end{array}$ \\
A tremendous number of round \\
cells, diffusely present in the whole \\
area of the choroid with focal \\
accumulation
\end{tabular}

cell population increased in number at 4 hours in some areas in the choroid and involved large areas of the choroid at 8 hours (Table 2).

The whole area of the choroid was infiltrated with a large number of round immunopositive (ED1) cells at 16 hours. At 24 hours after LPS injection, the round cells began to decrease in number, whereas the number of irregular cells and dendritiform cells increased. A striking feature, observed between 48 to 96 hours after LPS, was a large number of dendritiform cells, with more and longer dendrites than seen in untreated animals. A gradual decrease of both dendritiform cells and pleomorphic cells was noted at the later time points.

On day 14, the ED1 positive cells in the choroid returned to the preinjection levels both in number and in morphology (Table 2). The layer of macrophages bordering the sclera was not affected except for the occasional presence
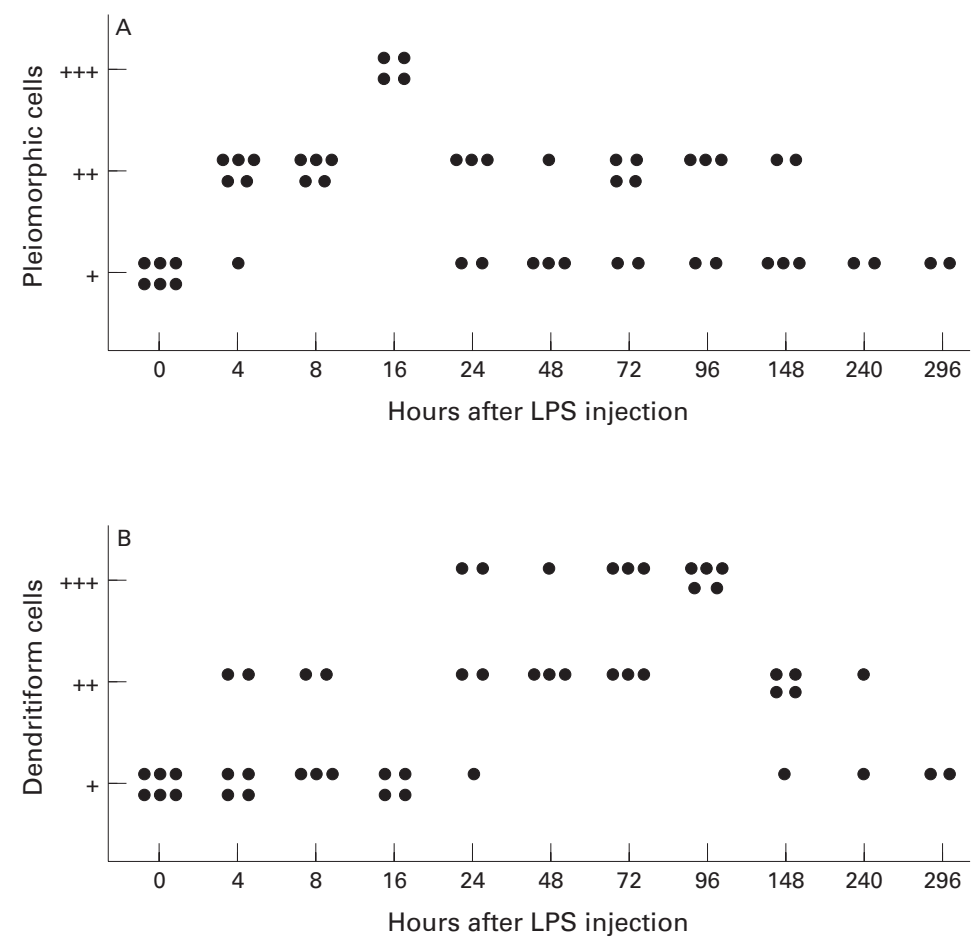

Figure 3 Dynamics of pleomorphic $(A)$ and dendritiform (B) ED1 positive cell numbers in the choroid after systemic LPS injection. The density of cells was scored as + (normal, low number), ++ (mild to moderate number), or +++ (high number). of a few round cells in some areas. Figure 3 shows the dynamics of ED1 positive cells in the choroid after LPS injection. The influx of pleomorphic cells was maximal at 16 hours after LPS injection, whereas the dendritiform cell number reached a maximum between 24 and 96 hours.

Staining for MHC class II showed an influx of immunostained round cells in the choroid at 8 hours. Subsequently, the number of these cells gradually increased and reached a peak at 72 and 96 hours (Fig 2). MHC class II positive dendritiform cells with longer and more dendrites compared with the cells observed in the normal choroid were detected at 72 and 96 hours. A gradual decrease of the MHC class II positive cells was noted at the later time points, returning to the preinjection level on day 14 . MHC class II positive cells were only found just beneath the RPE layer and LPS administration did not induce the appearance of these cells on the scleral side of the choroid.

\section{Discussion}

The present study confirms the presence of a network of tissue resident macrophages and MHC class II positive dendritic cells in the choroid of normal Lewis rats and shows that systemic LPS administration induces a marked temporary increase in both cell populations.

Immunohistological analysis of wholemounts, obtained from normal rats, revealed the presence of macrophages in a layer immediately below the RPE and in a layer bordering the sclera.

To our knowledge, the network of tissue resident macrophages on the outer surface of the choroid bordering the sclera has not yet been described. Our study revealed that the cells in this network are predominantly dendritiform cells and lack MHC class II membrane markers.

Previous studies using tissue sections showed the infiltration of the posterior segment of the eye by polymorphonuclear cells and mononuclear cells after LPS injection. ${ }^{78}$ These studies did not determine the phenotype of the infiltrating cells, nor did they evaluate the morphological features of the cells involved. The present study shows that systemic LPS injection induces a massive influx of macrophages into the choroid and emphasises the severe involvement of this tissue in EIU. The layer of macrophages bordering the sclera was not affected by LPS injection, a finding that was also confirmed by analysing tissue sections of this area (data not shown). It is not clear why the inner and outer layer of the choroid are not equally affected by LPS. It may be possible that LPS directly or indirectly stimulates RPE cells to release chemotactic cytokines which results in a preferential migration of inflammatory cells to the anterior side of the choroid. The migration of inflammatory cells into the choroid was seen as early as 4 hours after LPS injection and reached a maximum at 16 hours, which is similar to the invasion of the iris (unpublished own data). ${ }^{2-9}$ Moreover, a dramatic increase of dendritiform cells was noticed in the choroid between 48 to 96 hours 
after injection. Since there is no difference between the choroid and the anterior uvea with regard to the time course of the inflammatory response, it is reasonable to presume that the whole uvea is directly affected by LPS. Recently, we demonstrated a massive influx of ED1 positive cells into the retina during EIU but were not able to detect MHC class II positive cells, either before or after systemic LPS administration. ${ }^{3}$ Taken together these findings may also shed doubt on the concept that the EIU model is so 'eye' specific.

This latter finding contrasts with the choroid which contains a rich network of MHC class II positive cells. Our study using choroidal wholemounts, confirms earlier observations using cryostat sections whereby MHC class II positive dendritic cells were readily found in the rat choroid but not in the retina and sclera. ${ }^{10}$ Analysis of wholemounts revealed that the MHC class II positive dendritic cells were located in the choroid in close association with the RPE, which confirms earlier immunoelectron microscopic findings of Forrester et al. ${ }^{5}$ The number of MHC class II positive cells was significantly lower than that of ED1 positive cells. The exact nature of the infiltrating macrophages and dendritic cells was not analysed in our study. Whether LPS injection induces an influx in both macrophages and dendritic cells or whether only macrophages increase in number but now transiently express class II MHC warrants further study employing double label techniques. Enumeration of these cells first showed a peak of cells with a 'rounded pleomorphic' appearance at approximately 16 hours after LPS, followed by a rise in number of dendritiform cells occurring between 24 and 96 hours. These data strongly suggest an influx of blood borne round cells into the extravascular choroidal space followed by a transition of these cells into a dendritiform shape.

Double label experiments performed earlier by Forrester et $a l^{5}$ on choroidal sections revealed two distinct populations of cells since no double stained cells were observed when using OX-6 and ED2 monoclonal antibodies. Forrester et $a l^{5}$ found a smaller number of MHC class II positive cells in the choroid compared with the present data (373 versus 659 cells $/ \mathrm{mm}^{2}$ ). The reason for the discrepancy in these studies is unclear, but may be due to differences in the animals used, as has been pointed out earlier by McMenamin et al. ${ }^{6}$ In this study we focused on markers for dendritic cells and macrophages. EIU is also characterised by a heavy influx of polymorphonuclear cells whereas a role for $T$ lymphocytes is still a controversial issue. $^{29}$ (A de Vos et al, unpublished observations.)

MHC class II positive dendritic cells may play an important role in antigen presentation and initiation of an inflammatory response in the posterior segment of the eye. Isolation of MHC class II positive cells from the rat choroid and in vitro testing has confirmed that these cells have functional capacities in common with lymphoid dendritic cells with regard to $\mathrm{T}$ cell responses following mitogen stimula- tion or mixed lymphocyte reactions. ${ }^{10}$ The location of the choroidal MHC class II positive cells gives them ample opportunity to continuously sample antigens (foreign or self) from the choroidal vasculature or from the neighbouring RPE cells. Presentation of these antigens to T cells may initiate choroidal granulomatous disease as often seen in various uveitis entities and clinically represented by the appearance of fundal white dots. ${ }^{11}$

The concomitant presence of tissue resident macrophages, MHC class II positive dendritic cells, and RPE cells, each in close contact with each other, may represent a unique immunological microenvironment whereby the various cell types interact with each other to prevent overt choroidal inflammation. Disruption of this balance by, for instance, the release of bacterial endotoxin from sites elsewhere in the body may alter this balance. The consequences of an altered balance on the local control of immune responses are not yet known and deserves further investigation. The interaction of macrophages and dendritic cells has been extensively studied by Holt and co-workers, ${ }^{12}{ }^{13}$ who have shown a suppressive effect of resident macrophages in the lung on antigen presentation by dendritic cells. The suppressive role of macrophages pointed out by these authors refers to body cavity macrophages and it may well be that interstitial macrophages behave differently, owing to the fact that these latter cells may be proinflammatory by acting as a rich source of cytokines.

The short lived inflammation in the choroid as well as in the other ocular tissues, after systemic LPS injection, suggests that there may be potent suppressive mechanisms following the inflammatory response. Analysis of cytokine expression during EIU has shown a rapid induction of the interleukin-1 receptor antagonist, which is an important natural regulator of the cytokine network. ${ }^{14}$ Further elucidation of these and other anti-inflammatory mechanisms may provide a new understanding of the pathogenesis and treatment of human uveitis.

The authors thank Professor Dr C D Dijkstra of the Free University of Amsterdam for her kind gift of ED1, Mrs O F van Rossum for her help in typing the manuscript, and J de Feiter and P Hofmann for excellent technical assistance. P Yang was supported by a grant from the Guangdong Province Natural Science Foundation and the Foundation of the Health Ministry of the People's Republic of China.

1 Gery I, Streilein JW. Autoimmunity in the eye and its regulation. Curr Opin Immunol 1994;6:938-45.

2 Rosenbaum JT, McDevitt HO, Guss RB, Egbert PR. Endotoxin-induced uveitis in rats as a model for human Endotoxin-induced uveitis in rat
disease. Nature 19980;266:611-3.

3 Yang P, De Vos AF, Kijlstra A. Macrophages in the retina of normal Lewis rats and their dynamics after injection of lipopolysaccharide. Invest Ophthalmol Vis Sci 1996;37:7785.

4 Dijkstra CD, Dopp EA, Joling P, Kraal G. The heterogeneity of mononuclear phagocytes in lymphoid organs: distinct macrophage populations in the rat recognized by monoclonal antibodies ED1, ED2 and ED3. Immunology 1985;54:589-99.

5 Forrester JV, McMenamin PG, Holthouse I, Lumsden L, Liversidge $J$. Localization and characterization of major historid o i hility complex class II-positive cells in the istocompatibility comp posterior segment of the ef induction of autoimmune uveoretinitis. Invest Ophthalmol Vis Sci

6 McMenamin PG, Crewe J, Morrison S, Holt PG. Immunomorphologic studies of macrophages and MHC 
class II-positive dendritic cells in the iris and ciliary body of the rat, mouse and human eye. Invest Ophthalmol Vis Sci 4;35:3234-50

7 Okumura A, Mochizuki M. Endotoxin-induced uveitis in rats: morphological and biochemical study. Fpn $\mathcal{f}$ Ophthalmol 1988;32:457-65.

8 Ruiz-Moreno JM, Thillaye B, De Kozak Y. Retino-choroidal changes in endotoxin-induced uveitis in the rat. Ophthalmic Res 1992;24:162-8.

9 McMenamin PG, Crewe J. Endotoxin-induced uveitis: kinetics and phenotype of the inflammatory cell infiltrate and the response of the resident tissue macrophages and dendritic cells in the iris and ciliary body. Invest Ophthalmo Vis Sci 1995;36:1949-59.

10 Choudhury A, Pakalnis VA, Bowers WE. Characterization and functional activity of dendritic cells from rat choroid. Exp Eye Res 1994;59:297-304.
11 BenEzra D, Forrester JV. Fundal white dots: the spectrum of a similar pathological process. Br f Ophthalmol 1995;79: 856-60.

12 Holt PG, Schon-Hegrad MA, Oliver J. MHC class II antigen-bearing dendritic cells in the pulmonary tissues of the rat; regulation of antigen presentation activity by endogenous macrophage populations. F Exp Med 1988; 167:262-74.

13 Holt PG, Degebrodt A, O’Leary C, Krska K, Plozza T. T cell activation by antigen presenting cells from lung tissue digests: suppression by endogenous macrophages. Clin Exp Immunol 1985;62:586-93.

14 De Vos AF, Klaren VNA, Kijlstra A. Expression of multiple cytokines and IL-1RA in the uvea and retina during endotoxin-induced uveitis in the rat. Invest Ophthalmol Vis Sci 1994;35:3873-83. 